\title{
Violência Autoinfligida por Crianças e Adolescentes em um Município do Interior Paulista
}

\author{
Self-Inflicted Violence in Children and Teenagers in an Inland Town in São Paulo, Brazil \\ Violencia Autoinfligida por Niños y Adolescentes en una Ciudad del Interior de São Paulo, Brasil \\ Fernanda Perpétua Candido ${ }^{1}$ (D) https://orcid.org/0000-0003-1118-2789 \\ Maria Rita Rodrigues Vieira² (D) https://orcid.org/0000-0003-0103-0896 \\ Adriana Ferreira Rodrigues ${ }^{3}$ (D) htps://orcid.org/0000-0001-6075-3256
}

\section{Resumo}

Objetivo: Avaliar o perfil epidemiológico e a efetividade do acompanhamento de crianças e adolescentes atendidos em uma rede de atenção à saúde por violência autoprovocada.

Métodos: Foi realizado um estudo quantitativo, descritivo e retrospectivo por meio da análise de 177 fichas de notificação compulsória de violência interpessoal/autoprovocada e planilha de monitoramento online.

Resultados: A amostra foi majoritariamente composta por adolescentes do sexo feminino (77,40\%) entre 15 e 18 anos $(56,50 \%)$, com índice de reincidência elevado (45,20\%). Os principais locais de escolha identificados para abreviar a vida foram a residência $(85,31 \%)$ e a escola $(5,08 \%)$, tendo como meios mais utilizados 0 envenenamento/intoxicação (65,38\%) e o uso de objetos perfurocortantes $(26,37 \%)$.

Conclusão: 0 presente estudo evidenciou que adolescentes maiores de 15 anos, do sexo feminino, com deficiências/transtornos, e histórico de tentativas prévias apresentam risco aumentado para o suicídio, o que demonstra a necessidade de ferramentas mais efetivas para 0 acompanhamento das vítimas.

\section{Abstract}

Objective: To assess the epidemiological profile and the effectiveness of follow-up care with children and adolescents in healthcare service for self-inflicted violence.

Methods: A quantitative, descriptive and retrospective study was conducted on 177 compulsory notification records of interpersonal/self-inflicted violence and an online monitoring spreadsheet.

Results: The sample comprised mostly female adolescents $(77.4 \%)$ between 15 and 18 years of age $(56.5 \%)$ with a high relapse rate $(45.2 \%)$. The main locations chosen for the suicide attempts were the home $(85.31 \%)$ and the school (5.08\%), while the most common means were poisoning $(65.38 \%)$ and the use of cutting/piercing objects (26.37\%).

Conclusion: This study shows that female adolescents over 15, with disabilities/disorders, and a history of previous attempts are at a higher risk of suicide, demonstrating the need for more effective tools for monitoring victims.

\section{Resumen}

Objetivo: Evaluar el perfil epidemiológico y la efectividad del seguimiento de niños, niñas y adolescentes atendidos en una red de atención a la salud por violencia autoinfligida.

Métodos: Se realizó un estudio cuantitativo, descriptivo y retrospectivo mediante el análisis de 177 formularios de notificación obligatoria de violencia interpersonal 0 autoinfligida y una planilla de seguimiento online.

Resultados: La muestra estuvo compuesta mayoritariamente por mujeres adolescentes $(77,4 \%)$ entre 15 y 18 años $(56,5 \%)$ con una alta tasa de reincidencia $(45,2 \%)$. Los principales lugares elegidos para abreviar sus vidas fueron la residencia $(85,31 \%)$ y la escuela $(5,08 \%)$, y los medios más utilizados fueron el envenenamiento $(65,38 \%)$ y el uso de objetos cortantes $(26,37 \%)$.

Conclusión: El presente estudio mostró que adolescentes mujeres mayores de 15 años, con discapacidades/ trastornos y antecedentes de intentos previos tienen mayor riesgo de suicidio, lo que demuestra necesidad de desarrollar herramientas más efectivas para el seguimiento de las víctimas.

\section{Como citar:}

Candido FP, Vieira MR, Rodrigues AF. Violência Autoinfligida por Crianças e Adolescentes em um Município do Interior Paulista. Rev Soc Bras Enferm Ped. 2021;21(2):133-40.

${ }^{1}$ Associação Portuguesa de Beneficência de São José do Rio Preto, São José do Rio Preto, SP, Brasil.

${ }^{2}$ Faculdade de Medicina de São José do Rio Preto, São José do Rio Preto, SP, Brasil.

${ }^{3}$ Secretaria Municipal de Saúde de São José do Rio Preto, São José do Rio Preto, SP, Brasil.

Conflitos de interesse: nada a declarar.

Submetido: 28 de Setembro de 2021 | Aceito: 10 de Dezembro de 2021

Autor correspondente: Fernanda Perpétua Candido | E-mail: fernandacandido04@hotmail.com

Dol: http://dx.doi.org/10.31508/1676-379320210019 


\section{Introdução}

Segundo a Organização Mundial da Saúde (OMS), considera-se violência "o uso intencional de força física ou poder, real ou em ameaça, contra si próprio, contra outra pessoa, ou contra um grupo ou uma comunidade que resulte ou tenha possibilidade de resultar em lesão, morte, dano psicológico, deficiência de desenvolvimento ou privação". ${ }^{(1)}$ A OMS classifica a tipologia da violência em três grupos, segundo o autor do ato violento: violência destinada contra si (autoprovocada ou autoinfligida); violência interpessoal (doméstica e comunitária); e violência coletiva (grupos políticos, organizações terroristas e milícias). ${ }^{(1)}$

A Autolesão sem Intenção Suicida (ASIS) é caracterizada pelo ato de autoagressão contra si mesmo, compreende ações brandas e graves de automutilação e pode se apresentar sob a forma de cortes, arranhaduras, mordidas ou até mesmo a amputação de membros, demonstrando uma maneira de lidar com o sofrimento psíquico, mas sem o desejo de interromper a vida. ${ }^{(2,3)}$

A ideação suicida envolve um misto de pensamentos de autodestruição, que se configuram em planos que visam cessar a vida, antecedendo o suicídio. Embora não seja objeto de notificação, a ideação suicida configura-se em um forte fator de risco para o suicídio consumado, visto que, junto a ela, estão inclusas condições tais como a depressão e a descrença na vida e, portanto, requer ações de atenção integral. ${ }^{(4)}$

A autoagressão sem a consumação da morte é definida como Tentativa de Suicídio (TS), podendo gerar lesões incapacitantes, além de adoecimento do núcleo familiar. ${ }^{(5)} \mathrm{O}$ suicídio, por sua vez, advém do desejo consciente de morrer e de uma intenção clara e premeditada de provocar o ato, quando o viver torna-se insuportável. A ideação suicida, a tentativa de suicídio e o suicídio são três categorias do comportamento suicida. ${ }^{(4,5)}$

O suicídio ocorre em todos os ciclos da vida. Por ano, cerca de 900 mil pessoas morrem em decorrência deste, o equivalente a uma morte a cada 40 segundos e uma tentativa a cada três segundos. ${ }^{(6)}$ No ano de 2016, em nível mundial, foi dado como a segunda principal causa de morte entre jovens de 15 a 29 anos. ${ }^{(7)}$ Ainda que os números sejam preocupantes, é fato que ainda não demonstram a realidade, pois muitos casos ainda são subnotificados ou classificados erroneamente.
A infância e a adolescência são fases marcadas por constantes mudanças de ordem psicológica, física e/ou social, nas quais o indivíduo passa por diversos conflitos e ambivalências. Nesse momento, as crianças e os adolescentes também estão desenvolvendo estratégias para lidar com seus problemas existenciais, como compreender o sentido do viver e do morrer e, assim, as ideias suicidas podem aparecer como parte do processo, refletindo apenas a busca pela própria identidade. No entanto, a preocupação surge quando os pensamentos se tornam constantes e encarados como única alternativa para a resolução dos problemas. ${ }^{(4,8)}$

O percurso estabelecido entre a ideação e a concretização do suicídio oferece tempo oportuno para intervenção e, com base nestes fatores, essa pesquisa teve como objetivo avaliar o perfil epidemiológico e a efetividade do acompanhamento de crianças e adolescentes que se autoagrediram em um município do interior paulista.

\section{Métodos}

Foi realizado um estudo quantitativo, descritivo e retrospectivo a respeito do perfil epidemiológico e do acompanhamento de saúde de crianças e adolescentes que se autoagrediram em 2018 no município de São José do Rio Preto - SP. Os resultados foram obtidos por meio da análise de fichas de notificação compulsória de Violência Interpessoal/Autoprovocada, inseridas no banco de dados do Sistema de Informação de Agravos de Notificação (SINAN). Além disso, foi avaliada a planilha de monitoramento online, disponibilizada pela área técnica de Doenças e Agravos não Transmissíveis (DANT), no setor de Vigilância Epidemiológica da Secretaria de Saúde do referido município.

A inserção das fichas nos sistemas de informação ocorre após o preenchimento pelos serviços responsáveis (unidades de saúde, secretarias de assistência social, e educação), sendo posteriormente qualificadas pelo profissional responsável. A inclusão das fichas no SINAN é realizada pela Gerência de Informação de Vigilância em Saúde (GIVS), também situada na Secretaria Municipal de Saúde.

Após recebimento e qualificação das fichas, o profissional verifica o histórico de atendimento da vítima 
através do sistema Empro Saúde (responsável por interligar os serviços públicos de saúde) e, em seguida, encaminha um e-mail para a gerência da unidade básica de saúde de referência da vítima, comunicando a inserção de um novo caso e alertando para que a busca ativa e o acompanhamento sejam feitos.

O preenchimento da planilha no campo de acompanhamento é de responsabilidade do serviço que o realiza, ou seja, das Unidades de Atenção Básica (UBS/ UBSF) e/ou Especializada (CAPSi). A planilha de monitoramento foi criada em 2017 e atua como uma ferramenta online (Google Drive) de comunicação entre os dispositivos de saúde responsáveis pelo acompanhamento das vítimas.

Para cumprir com os objetivos propostos, foram coletadas informações de 177 registros, nos quais foi feita a avaliação das seguintes variáveis: idade; sexo; raça/cor; gestante; escolaridade; presença de deficiência/transtorno; tipo de deficiência/transtorno (quando presente); local de ocorrência; período do dia; suspeita de uso de álcool; reincidência; meio de agressão; relato do meio de agressão; atendimento em outros serviços de saúde; e segmento de saúde/ acompanhamento. Após a tabulação dos dados coletados, foram aplicadas duas análises estatísticas: a descritiva e a inferencial.

Para a análise estatística descritiva, foi traçado o perfil da amostra estudada, contemplando as variáveis propostas, de forma que os dados foram apresentados de forma absoluta e relativa por meio de tabelas. Além disso, foi possível caracterizar o grupo amostral com relação à faixa etária média, desvio padrão, valor máximo e valor mínimo.

Para a análise estatística inferencial, inicialmente, foi aplicado o teste de Komolgorov-Smirnov, a fim de verificar a normalidade dos dados. Para verificar a correlação entre as variáveis, na qual se parametriza uma das variáveis como sendo dependente e a outra como independente, objetivando a análise de predição entre ambas, utilizou-se o teste U de Mann-Whitney. O nível de significância considerado foi de $5 \%(p<0,05)$ e todos os testes contemplam erro alfa de $5 \%$ e confiabilidade de $95 \%$. Todas as análises foram obtidas a partir do Software SPSS Statistics (Versão 23), atreladas às funcionalidades do Excel (versão 2016).

É importante relatar que houveram perdas de dados na avaliação das variáveis, tanto nas fichas de notificação como na planilha de monitoramento, devido a eventuais erros de preenchimento e/ou incompletude de dados. Alguns exemplos de falhas que ocorreram foram: quadrículos não preenchidos e/ou preenchidos erroneamente nas fichas; inserção na planilha de monitoramento em locais inadequados (regiões, unidades de saúde de referência, e grupo etário); ou digitação errônea dos dados identificadores (nome completo, idade). Tais perdas de dados foram classificadas como "Missing" durante a coleta e análise dos dados.

A realização do estudo ocorreu somente após a autorização da Comissão de Pesquisa Científica da Secretaria Municipal de Saúde de São José do Rio Preto e do Comitê de Ética em Pesquisa (CEP) da Faculdade de Medicina de São José do Rio Preto - SP (CAAE: 39130720.6.0000.5415 - Parecer no 4.411.540). A coleta de dados, cuja análise foi efetuada em caráter retrospectivo, foi executada por meio de um banco de dados nacional e uma planilha de dados municipais, dispensando, assim, o Termo de Consentimento Livre Esclarecido (TCLE), pois a segurança e o anonimato dos dados e das vítimas foram devidamente assegurados.

\section{Resultados}

Foram analisadas 177 fichas de notificação compulsória de Violência Interpessoal/ Autoprovocada referentes ao ano de 2018, consultadas no banco de dados do SINAN e planilha de monitoramento online, na faixa etária compreendida entre $01 \mathrm{e}$ 18 anos de idade. A partir dos dados coletados foi possível verificar que a idade média contida nas notificações foi de 15,38 anos $( \pm 2,03)$, com idade máxima e mínima de 18 e 8 anos, respectivamente. As notificações ocorreram predominantemente na faixa etária correspondente aos indivíduos maiores de 15 anos (56,50\%) do sexo feminino (77,40\%). Em relação à raça e cor, as autoagressões ocorreram predominantemente nas crianças e adolescentes de cor branca $(70,06 \%)$ e foi identificada apenas uma gestação em curso $(0,56 \%)$ entre as notificações. A ideação suicida entre os jovens com ensino médio incompleto representa o maior índice da amostra $(31,64 \%)$ (Tabela 1$)$. 
Tabela 1. Dados de identificação pessoal, segundo registro nas fichas de notificação compulsória

\begin{tabular}{lc}
\hline Variáveis & $\mathrm{n}(\%)$ \\
\hline Idade & \\
Até 10 anos & $2(1,13)$ \\
11 a 15 anos & $75(42,37)$ \\
$>15$ anos & $100(56,50)$ \\
Sexo & \\
Feminino & $137(77,40)$ \\
Masculino & $40(22,60)$ \\
Raça/cor & \\
Branca & $124(70,06)$ \\
Preta & $9(5,08)$ \\
Parda & $41(23,16)$ \\
Indígena & $1(0,56)$ \\
Missing & $2(1,13)$ \\
Gestante & \\
$2^{0}$ Trimestre & $1(0,56)$ \\
Não & $124(70,06)$ \\
Não se aplica & $42(23,73)$ \\
Missing & $10(5,65)$ \\
Escolaridade & \\
$1^{\text {a a } 4}{ }^{\text {a }}$ série incompleta do EF & $7(3,95)$ \\
$4^{\text {a }}$ série completa do EF & $2(1,13)$ \\
$5^{\text {a }}$ a $8^{\text {a }}$ série incompleta do EF & $46(25,99)$ \\
Ensino fundamental completo & $10(5,65)$ \\
Ensino médio incompleto & $56(31,64)$ \\
Ensino médio completo & $24(13,56)$ \\
Não se aplica & $1(0,56)$ \\
Missing & $31(17,51)$ \\
Total & $177(100,00)$ \\
\hline & \\
\hline & \\
\hline
\end{tabular}

A presença de deficiência/ transtorno foi constatada em $35,59 \%$ dos indivíduos, nos quais preponderou o transtorno de comportamento $(18,72 \%)$. Para a variável referente ao tipo de deficiência, o número total ultrapassou 177 , devido à possibilidade de preenchimento de mais de uma opção nas fichas (Tabela 2).

Tabela 2. Dados referentes à presença de deficiência/ transtorno, segundo registro nas fichas de notificação compulsória

\begin{tabular}{lc}
\hline Variáveis & $\mathbf{n}(\%)$ \\
\hline Possui algum tipo de deficiência/transtorno? & \\
Não & $96(54,24)$ \\
Sim & $63(35,59)$ \\
Missing & $18(10,17)$ \\
Total & $177(100,00)$ \\
Se sim, qual tipo? & \\
Deficiência intelectual & $3(1,60)$ \\
Transtorno mental & $29(15,51)$ \\
Transtorno de comportamento & $35(18,72)$ \\
Outras & $6(3,21)$ \\
Não se aplica & $114(60,96)$ \\
Total & $187(100,00)$ \\
\hline
\end{tabular}

A residência foi o local de escolha predominante para a tentativa do ato suicida por crianças e adolescentes $(85,31 \%)$, seguido da escola $(5,08 \%)$. Com relação ao período do dia em que ocorreu a tentativa de suicídio, a noite $(35,03 \%)$ e a tarde $(19,77 \%)$ foram predominantes. Para uma pequena porcentagem da amostra $(4,25 \%)$ foi relatada suspeita do uso de álcool. Além disso, foram encontradas reincidências em $45,20 \%$ das notificações registradas (Tabela 3).

Tabela 3. Dados referentes à ocorrência da violência, segundo registro nas fichas de notificação compulsória

\begin{tabular}{lc}
\hline Variáveis & $\mathrm{n}(\%)$ \\
\hline Local de ocorrência & $151(85,31)$ \\
Residência & $3(1,69)$ \\
Habitação coletiva & $9(5,08)$ \\
Escola & $7(3,95)$ \\
Via pública & $7(3,95)$ \\
Missing & \\
Período do dia & $15(8,47)$ \\
Manhã (06:00-11:59) & $35(19,77)$ \\
Tarde (12:00-17:59) & $62(35,03)$ \\
Noite (18:00-23:59) & $15(8,47)$ \\
Madrugada (00:00-5:59) & $50(28,25)$ \\
Missing & \\
Suspeita de uso de álcool? & $139(78,53)$ \\
Não & $8(4,52)$ \\
Sim & $30(16,95)$ \\
Missing & \\
Reincidência & $73(41,24)$ \\
Não & $80(45,20)$ \\
Sim & $24(13,56)$ \\
Missing & $177(100,00)$ \\
\hline
\end{tabular}

O meio de agressão mais utilizado foi o de envenenamento/ intoxicação $(65,38 \%)$, dentre os quais a ingestão de medicações foi a mais frequente $(36,72 \%)$ e com associação a outros meios $(40,7 \%)$, conforme relatos na planilha de monitoramento. $\mathrm{O}$ segundo meio de agressão mais utilizado envolveu o uso de objetos perfurocortantes $(26,37 \%)$, dentre os quais $14,69 \%$ representaram a escolha do objeto isoladamente, enquanto $15,81 \%$ ocorreram em associação com outros meios. Vale salientar que, para as informações relativas ao meio de agressão, o número total ultrapassou 177 , devido à possibilidade de preenchimento de mais de um quadrículo nas fichas (Tabela 4).

Em relação ao atendimento, verificou-se que $15,25 \%$ das vítimas necessitaram de atendimento em outros serviços, sendo direcionadas, em sua maioria, 
Tabela 4. Dados referentes ao meio de agressão utilizado, segundo registro nas fichas de notificação compulsória e planilha de monitoramento online

\begin{tabular}{lc}
\hline Variáveis & $\mathrm{n}(\%)$ \\
\hline Meio de agressão & \\
Enforcamento & $5(2,75)$ \\
Objeto contundente & $4(2,20)$ \\
Objeto perfurocortante & $48(26,37)$ \\
Envenenamento/intoxicação & $119(65,38)$ \\
Ameaça & $5(2,75)$ \\
Outro & $1(0,55)$ \\
Total & $182(100,00)$ \\
Relato do meio de agressão & \\
Autoagressão & $1(0,56)$ \\
Autoagressão com arranhões & $1(0,56)$ \\
Autoagressão com objeto contundente & $1(0,56)$ \\
Autoagressão com objeto perfurocortante & $26(14,69)$ \\
Autoagressão com objeto perfurocortante/contundente & $1(0,56)$ \\
Ingesta de medicações & $65(36,72)$ \\
Ingesta de pesticidas & $1(0,56)$ \\
Ingesta de pesticidas/álcool & $1(0,56)$ \\
Ingesta de medicações/álcool & $2(1,13)$ \\
Ingesta de medicações/ autoagressão com objeto & $1(0,56)$ \\
perfurocortante & \\
Ingesta de medicações/saneantes domissanitários & $4(2,26)$ \\
Ingesta de saneantes domissanitários & $3(1,69)$ \\
Ingesta de vidro & $2(1,13)$ \\
Tentativa de atirar-se de uma ponte & $1(0,56)$ \\
Tentativa de atirar-se em frente a carros & $1(0,56)$ \\
Tentativa de enforcamento & $3(1,69)$ \\
Tentativa de se jogar de um lugar elevado & $1(0,56)$ \\
Missing & $62(35,05)$ \\
Total & $177(100,00)$ \\
\hline
\end{tabular}

para a emergência psiquiátrica. A variável "segmento de saúde - acompanhamento", cujo número total ultrapassou 177, ocorreu devido à possibilidade do preenchimento de mais de um campo na planilha de monitoramento. A respeito do segmento de saúde em que as vítimas foram acompanhadas, a Atenção Básica/ NASF foi a responsável pela maioria dos atendimentos $(44,15 \%)$, conforme pode ser observado na tabela 5 .

Tabela 5. Dados referentes ao atendimento/ acompanhamento de saúde, segundo registro na planilha de monitoramento online

\begin{tabular}{lc}
\hline Variáveis & $\mathrm{n}(\%)$ \\
\hline Atendimento em outros serviços de saúde? & \\
Sim & $27(15,25)$ \\
Missing & $150(84,75)$ \\
Total & $177(100,00)$ \\
Segmento de saúde - acompanhamento & \\
Atenção básica/ NASF & $83(44,15)$ \\
Saúde mental (CAPSi) & $19(10,11)$ \\
Missing & $86(45,74)$ \\
Total & $188(100,00)$ \\
\hline
\end{tabular}

Foi realizada uma análise inferencial de correlação estatística entre as variáveis, na qual foi possível revelar a existência de diferenças significativas $(p<0,05)$, comprovando a relação entre elas. Nesse sentido, verificou-se associações entre: período e local de ocorrência $(p=0,009)$; período e idade $(p=0,038)$; e reincidência e presença de deficiência/ transtorno $(p=0,001)$. Com relação à associação entre o período do dia e o local de ocorrência, verificou-se que a maioria das autoagressões ocorrem durante a noite na residência da vítima. Por outro lado, as tentativas de suicídio que ocorreram na escola foram predominantemente durante o período da manhã e da tarde. Para a associação entre o período do dia e a idade, verificou-se que a maior parte das notificações de jovens entre 11 e 15 anos e maiores de 15 anos ocorreram no período noturno. Por fim, houve uma correlação significativa entre as notificações de reincidência e a presença de deficiência/ transtorno, evidenciando que tal condição pode ser considerada um fator de risco para tentativas de suicídio futuras.

\section{Discussão}

A tentativa de suicídio em indivíduos maiores de 15 anos confirma a prevalência aumentada de tal ideação na adolescência. Por se tratar de um período de transição, em que o indivíduo se encontra fragilizado, torna-se susceptível ao comportamento suicida em resposta às desordens que vive, tanto de ordem pessoal, quanto social, como, por exemplo, ao questionar sobre a sua sexualidade, possuir um histórico de abuso sexual, a ocorrência de transtornos psiquiátricos em investigação e o diagnóstico de estresse pós-traumático. ${ }^{(9)}$

Outro importante fator de risco é a reprodução de comportamentos midiáticos, mais prevalente em adolescentes do que em adultos, e que representa um risco potencial para causar epidemias de suicídios. ${ }^{(9)}$ As redes sociais são, por vezes, o maior meio de socialização entre os adolescentes, devido ao seu fator positivo de distração e entretenimento. No entanto, o espaço virtual pode se tornar perigoso, pois, ao passo que o internauta procura por autoajuda, também pode fazer o contrário, ou seja, de qualquer forma, obterá resposta para o que procura. ${ }^{(10)}$ 
A internet também pode ser responsável por gerar gatilhos e impulsionar tentativas de suicídio, como, por exemplo, com a divulgação indevida de conteúdo privado, passível de gerar transtorno pessoal, familiar e/ou social. Outros exemplos comuns são: o bullying virtual e os jogos online. ${ }^{(10)}$ Alguns jogos online podem colocar a vida de seus participantes em risco, tais como: o jogo do desmaio, que implica em manter apneia intencional até atingir a inconsciência; o jogo da fada, que incentiva crianças e adolescentes a ligarem o botijão de gás de cozinha durante a noite e sem o conhecimento dos pais; e o jogo da baleia azul, que é constituído por 50 desafios autodestrutivos, sendo o último destinado a tirar a própria vida. ${ }^{(10)}$

O sexo feminino foi prevalente nas notificações avaliadas, corroborando com outros estudos que também apontaram um risco aumentado do comportamento suicida para mulheres. Embora o sexo feminino tenha a característica de reincidir mais nas tentativas, a consumação da intenção é mais prevalente no sexo masculino. ${ }^{(11)}$ No ano de 2006, a proporção de óbitos por suicídio do sexo masculino em relação ao feminino foi de 5,4 para 1. Provavelmente, isso ocorre devido à dificuldade da exteriorização do sofrimento psíquico associado ao sexo masculino, o que dificulta o diagnóstico de possíveis quadros depressivos. ${ }^{(9)}$

Quanto à raça/ cor, o número de indivíduos de cor branca notificados sobressaiu-se aos demais, resultado também apresentado por outros estudos. No entanto, pesquisadores afirmam que não há relação entre tais variáveis, visto que o suicídio independe desses fatores e está relacionado às sensações e experiências de cada um com o viver, tanto consigo, como na família e na sociedade. ${ }^{(12)}$

Com relação à presença de gestação em curso, verificou-se uma ocorrência baixa, o que pode ser explicado pelo fato de a maternidade atuar como um fator protetivo contra o suicídio, principalmente nos anos consecutivos ao parto, pois as mães passam a enxergar em si um novo papel social, em que assumem certo protagonismo na vida dos filhos. Todavia, nos casos em que não há uma rede de apoio sustentável, associada à presença de transtornos médicos ou outros fatores de risco, essa condição pode se tornar uma vulnerabilidade. ${ }^{(13)}$

No que se refere à escolaridade, as notificações de indivíduos com ensino médio incompleto prevalece- ram, o que relaciona-se diretamente à faixa etária mais prevalente na amostra. Contudo, ainda que a baixa escolaridade se associe a ideação suicida, isoladamente, não predispõe e nem impede o suicídio. ${ }^{(12)}$

Além disso, foi identificada a presença de transtorno mental e de transtorno de comportamento em uma parcela significativa da amostra, dado que corrobora com outros estudos que trazem essas condições como fatores de risco para o comportamento suicida, principalmente quando não diagnosticadas e não tratadas. ${ }^{(4,5)}$

Os principais locais de escolha para a consumação do comportamento suicida foram a residência e a escola e isso se deve ao fato de serem locais de livre acesso, nos quais a faixa etária considerada perpassa mais tempo. Cumpre salientar que o monitoramento adequado e a sensibilidade para identificar os fatores de risco que contribuem para tal prática é uma tarefa importante a ser desenvolvida pelos responsáveis legais e pelos profissionais da educação. ${ }^{(14)}$

As tentativas de suicídio deram-se, principalmente, nos períodos vespertino e noturno. Um estudo realizado em uma unidade de emergência evidenciou que a maioria dos atendimentos ocorria no período noturno, contrariamente aos boletins de ocorrência policial, que ocorriam no período diurno, com o horário real da tentativa. Nessa pesquisa em específico, os autores verificaram que a maioria dos adolescentes ficavam sozinhos em casa durante o período diurno sem supervisão, com tempo hábil para praticar tais tentativas. $^{(15)}$

O uso de álcool foi identificado em uma pequena porcentagem na amostra, o que pode ser explicado pelo fato da venda ou qualquer meio de fornecimento de bebidas alcoólicas para menores de 18 anos de idade ser crime no Brasil. ${ }^{(16)}$ Apesar disso, os meios de fiscalização são escassos e a mídia incentiva diariamente o seu uso.

O número de reincidentes evidenciado foi preocupante, assim, observou-se que uma tentativa não consumada acaba levando a outras, caso não haja intervenção. As maiores chances para os indivíduos reincidirem ocorrem geralmente após três meses a um ano da primeira tentativa, podendo ser fatal. Estima-se ainda que, para cada tentativa registrada, existem outras quatro não registradas, devido à subnotificação dos serviços responsáveis pelo atendimento dessas vítimas. Além disso, muitas tentativas também acabam 
não chegando até o serviço de atendimento em saúde, por serem de menor gravidade. ${ }^{(17)}$

Em sua maioria, as autoagressões deram-se por envenenamento/ intoxicação, devido à facilidade de acesso e ao descuido no armazenamento de medicações, pois a automedicação é um hábito cultural da população brasileira, e que, muitas vezes, leva à criação de um estoque de fármacos no domicílio, configurando a chamada "farmácia caseira". Por se tratar do meio de menor letalidade, está também diretamente associado às reincidências, podendo predispor os indivíduos não assistidos a desenvolver métodos mais eficazes para a consumação do ato. ${ }^{(18)}$

Em segundo plano, o uso de objetos perfurocortantes obteve como um dos meios mais utilizados para a autoagressão. Estudos demonstram, em nível mundial, que essa prática tem se tornado crescente entre os adolescentes, o que é um fator preocupante, pois configura um risco aumentado para o suicídio. $\mathrm{O}$ ato de mutilar a própria pele por meio de objetos cortantes expressa o desejo de aliviar algum sentimento ou tensão emocional, mas sem intenção suicida consciente. ${ }^{(19)}$

A Atenção Básica/ NASF foi responsável pela maior parte dos acompanhamentos e o seu papel pode ser considerado como crucial na prevenção de tentativas de suicídios. Um estudo internacional, feito a partir da análise de 40 artigos científicos em seis países, evidenciou que $45 \%$ dos que morreram por suicídio foram atendidos na Atenção Primária à Saúde (APS), cerca de 30 dias antes da sua morte. No ano que antecedeu o suicídio, $32 \%$ das vítimas foram atendidas em serviços de saúde especializada, enquanto $77 \%$ foram atendidas na APS. ${ }^{(20)}$ Uma pequena porcentagem da amostra recebeu atendimento inicial em outros serviços de saúde que não na Unidade de Atenção Básica (UBS/UBSF) ou Unidades de Pronto Atendimento (UPAs). Vale salientar que a maior parte das fichas não foram encontradas ou não tiveram o campo em questão preenchido na planilha de monitoramento online.

O que define o correto encaminhamento da vítima é a avaliação do risco de suicídio, estratificado em alto, moderado e baixo. Se o risco for alto: indivíduo com histórico de tentativas prévias, ideação suicida frequente, planejamento e acesso aos meios de consumação, deve ser encaminhado imediatamente para emergência psiquiátrica. Se o risco for moderado: indivíduo com histórico de reincidência, ideação suicida persistente, sem planejamento do ato, deve ser feita uma avaliação imediata para verificar se há critério de internação ou acompanhamento intensivo em Centro de Atenção Psicossocial (CAPS). Se o risco for baixo: indivíduo sem histórico de reincidência e planejamento, mas com ideação suicida, o cuidado deve ser mantido na APS, com monitoração constante da evolução da ideação. ${ }^{(20)}$

Em São José do Rio Preto, interior paulista, as portas de entrada do serviço de saúde pública para atendimentos desse agravo são as da Atenção Básica (UBS/ UBSF) e das Unidades de Pronto Atendimento (UPAs), ambas responsáveis pelo primeiro atendimento e acolhimento da vítima. Ao se tratar de um caso agudo, com alto risco e necessidade de internação, deve ser acionado o Serviço de Atendimento Móvel de Urgência (SAMU) para direcionamento ao hospital psiquiátrico do município (Hospital Bezerra de Menezes). Já os casos moderados e leves são todos atendidos com prioridade e encaminhados para atenção especializada, o CAPSi.

\section{Conclusão}

O presente estudo evidenciou que adolescentes maiores de 15 anos apresentam risco aumentado para o suicídio. Foram identificados como fatores de risco: sexo feminino; presença de deficiências/transtornos; e histórico de tentativas prévias. A maioria das autoagressões deram-se em ambientes culturalmente considerados seguros (residência e escola). Quanto ao atendimento/acompanhamento de saúde, no geral, a planilha de monitoramento online foi eficaz e colaborativa com o processo de trabalho, mas dependente de aparatos complementares. As esferas municipais envolvidas na atenção integral do perfil epidemiológico evidenciado pelo presente estudo, bem como os responsáveis legais, educadores, e profissionais da saúde, devem se sensibilizar quanto a temática para agir precocemente na detecção e controle dos fatores de risco identificados. A exemplo de algumas medidas preventivas/ protetivas, que devem ser amplamente disseminadas, é possível destacar: condicionamento adequado de medicações e objetos perfurocortantes em escolas e residências; supervisão do uso do material escolar; identificação precoce e tratamento de deficiências/ 
transtornos; e identificação e controle de outros fatores de risco (comportamentos influenciados por conteúdo midiático e o uso de substâncias lícitas e ilícitas). Além disso, é preciso salientar a importância de haver um constante aprimoramento e capacitação dos profissionais da saúde, para elaboração e implementação de novas ferramentas, bem como o uso adequado das existentes. No caso das fichas de notificação, há um documento online e autoexplicativo disponibilizado pelo Ministério da Saúde (Viva - instrumento de notificação de violência interpessoal e autoprovocada), o qual deve ser explorado pelas unidades de saúde para permitir o preenchimento adequado das mesmas. Quanto a planilha de monitoramento, recomenda-se que seja reformulada, de modo a restringir-se apenas às informações necessárias, com atenção especial para a isenção dos dados identificadores e preenchimento dos campos de acompanhamento. Portanto, conclui-se que dados fidedignos e de qualidade demandam ferramentas, meios de aplicação, e recursos humanos favoráveis e adequados, assim, para garantir tais premissas básicas, sugere-se o desenvolvimento de práticas constantes e conjuntas entre as esferas envolvidas, com o intuito de reduzir as possíveis lacunas de atendimento às vítimas de autoagressão.

\section{Contribuições}

Candido FP, Vieira MRR e Rodrigues AF declaram que contribuíram com a concepção do projeto, análise e interpretação dos dados, revisão crítica relevante do conteúdo intelectual e aprovação da versão final a ser publicada.

\section{Referências}

1. Brasil. Ministério da Saúde. VIVA: Instrutivo de notificação de violência interpessoal e autoprovocada. Brasília (DF): Ministério da Saúde; 2016 [citado 2021 Jul 20]. Disponível em: http://bvsms.saude.gov.br/bvs/publicacoes/viva_instrutivo_violencia_ interpessoal_autoprovocada_2ed.pdf
2. Brasil. Senado Federal. CPI dos maus-tratos contra crianças e adolescentes: vamos conversar sobre a prevenção da automutilação? Brasília (DF): Senado Federal; 2017 [citado 2021 Set 07]. Disponível em: http://www.crianca.df.gov.br/wp-conteudo/ uploads/2018/04/Vamos-Conversar-sobre-prevencao-da-automutilaca02.pdf

3. Bahia CA,Avanci JQ, Pinto LW, Minayo MC. Lesão autoprovocada em todos os ciclos da vida: perfil das vítimas em senviços de urgência e emergência de capitais do Brasil. Ciênc Saúde Colet. 2017;22(9):2841-50.

4. Moreira LC, Bastos PR. Prevalência e fatores associados à ideação suicida na adolescência: revisão de literatura. Psicol Esc Educ. 2015;19(3):445-53.

5. Braga LL, Dell'Aglio DD. Suicídio na adolescência: fatores de risco, depressão e gênero. Contextos Clínic. 2013;6(1):2-14.

6. Rosa NM, Agnolo CM, Oliveira RR, Mathias TA, Oliveira ML. Tentativas de suicídio e suicídios na atenção pré-hospitalar. J Bras Psiquiatr. 2016;65(3):231-8.

7. Organização Pan-Americana da Saúde (OPAS). Suicídio. Brasília (DF): Escritório Regional para as Américas da Organização Mundial da Saúde; 2018 [citado 2021 Jul 23]. Disponível em: https://www.paho.org/bra/index.php?option=com_content\&view=article\&id=5671:f olha-informativa-suicidio\&ltemid $=839$

8. Fernandes VM, Zacharias DG. Adolescência, suicídio e redes de apoio: 0 desejo de morte enquanto grito pela vida. Santa Cruz do Sul: Anais da V Jornada de Pesquisa em Psicologia: diálogos interdisciplinares; 2015 [citado 2021 Jul 29]. Disponível em: http://online.unisc. br/acadnet/anais/index.php/jornada_psicologia/article/view/14516

9. Cicogna J, Hillesheim D, Hallal AL. Mortalidade por suicídio de adolescentes no Brasil: tendência temporal de crescimento entre 2000 e 2015. J Bras Psiquiatr. 2019;68(1):1-7.

10. Junior IF, Lima MA. Suicídio e o jogo da baleia azul analisados na perspectiva de anomia de Émile Durkheim. Rev Sociol Antropol Cult Juríd. 2017;3(1):121-36.

11. Tirolla RM, Girotto E, Guidoni CM. Análise clínica e epidemiológica das tentativas de suicídio em crianças atendidas em um centro de informação e assistência toxicológica. Rev Paul Pediatr. 2020;39:1-6.

12. Schlichting CA, Moraes MC. Mortalidade por suicídio na adolescência: uma revisão. Rev Fam Ciclos Vida Saúde Contexto Soc. 2018; 6(1):357-63.

13. Alpe AC, Alf AM. Significados atribuídos ao comportamento suicida por adolescentes do sexo feminino. Estud Interdiscip Psicol. 2020;11(3):99-115.

14. Manei SS. Perfil epidemiológico das tentativas de suicídio em crianças e adolescentes registradas no centro de informação e assistência toxicológica de Santa Catarina, no período de janeiro de 2013 a dezembro de 2017: 0 que mudou em 10 anos? Florianópolis: Universidade Federal de Santa Catarina; 2019 [citado 2021 Set 08]. Disponível em: https://repositorio.ufsc.br/handle/123456789/203319

15. Avanci RC, Pedrão ப, Costa Júnior ML. Perfil do adolescente que tenta suicídio em uma unidade de emergência. Rev Bras Enferm. 2005;58(5) 535-9.

16. Senado Notícias. Câmara aprova tipificação do crime de venda de bebida alcoólica a menor de 18 anos. Brasília (DF): Agência Senado; 2015 [citado 2021 Set 18]. Disponível em: $\quad$ https://www12.senado.leg.br/noticias/materias/2015/02/25/camara-aprovatipificacao-do-crime-de-venda-de-bebida-alcoolica-a-menor-de-18-anos

17. Gomes MS, Costa TD. Tentativas de suicídio de crianças e adolescentes em Sergipe no período de 2015 a 2017. São Cristóvão: Universidade Federal de Sergipe; 2018 [citado 2021 Set 15]. Disponível em: https://ri.ufs.br/handle/riufs/12242

18. Kitagawa T, Sales CC, Paiano M, Oliveira ML. Adolescentes internados por tentativa de suicídio com agentes químicos: um estudo transversal. Semin Ciênc Biol Saúde. 2019;40(1):5-14.

19. Carmo JS, Silveira PH,Vignardi RG, Canicoba GS, Mota AC, Miziara CS, et al.Autolesão não suicida na adolescência como fator de predisposição ao suicídio. Saúde Ética Justiça. 2020;25(1):3-9.

20. Secretaria Municipal de Saúde (SMS). Coleção Guia de Referência Rápida: Avaliação do Risco de Suicídio e sua Prevenção. Rio de Janeiro: Série F. Comunicação e Educação em Saúde; 2016 [citado 2021 Set 03]. Disponível em: https://subpav.org/download/prot/ Guia_Suicidio.pdf 